\title{
Electronic Raman response in electron-doped cuprate superconductors
}

\author{
Zhihao Geng and Shiping Feng \\ Department of Physics, Beijing Normal University, Beijing 100875, China
}

\begin{abstract}
The electronic Raman response in the electron-doped cuprate superconductors is studied based on the $t-t^{\prime}-J$ model. It is shown that although the domelike shape of the doping dependent peak energy in the $B_{2 g}$ symmetry is a common feature for both electron-doped and hole-doped cuprate superconductors, there are pronounced deviations from a cubic response in the $B_{1 g}$ channel and a linear response in the $B_{2 g}$ channel for the electron-doped case in the low energies. It is also shown that these pronounced deviations are mainly caused by a nonmonotonic d-wave gap in the electron-doped cuprate superconductors.
\end{abstract}

PACS numbers: 74.25.nd, 74.72.Ek, 74.20.Mn, 74.20.-z

The parent compounds of cuprate superconductors are believed to belong to a class of materials known as Mott insulators with an antiferromagnetic (AF) long-range order (AFLRO), where a single common feature is the presence of the $\mathrm{CuO}_{2}$ plane [1, 2]. As the $\mathrm{CuO}_{2}$ planes are doped with charge carriers, holes or electrons, the $\mathrm{AF}$ phase subsides and superconductivity emerges [1, 2]. It has been found that only an approximate symmetry in the phase diagram exists about the zero doping line between the hole-doped ( $p$-doped) and electron-doped ( $n$ doped) cuprate superconductors, and the significantly different behavior of the $p$-doped and $n$-doped cuprate superconductors is observed [3], reflecting the electron-hole asymmetry. For the $p$-doped cuprate superconductors, AFLRO is reduced dramatically with doping, and vanished around the doping $\delta \sim 0.05$, then superconductivity emerges over a wide range of the hole doping concentration $\delta$, around the optimal doping $\delta \sim 0.15$ [1, 3], where the pair-breaking peaks observed from the Raman scattering measurements have been extracted in different polarization configurations [4 8], and the results show that the electronic Raman response (ERR) depends linearly on energy in the $B_{2 g}$ channel in the low energy limit, and depends cubically on energy in the $B_{1 g}$ channel. However, AFLRO survives until superconductivity appears over a narrow range of the electron doping concentration $\delta$, around the optimal doping $\delta \sim 0.15$ in the $n$-doped counterparts [2, 3], where although the low-energy behaviors of the $B_{1 g}$ and $B_{2 g}$ channels approach very specific power laws consistent with the presence of lines nodes in the superconducting (SC) gap, the pair-breaking peak energy values in the $B_{1 g}$ and $B_{2 g}$ channels can be different in some instances, while in some others they are virtually identical 9 -13]. In this case, the investigating similarities and differences of ERR between the $p$-doped and $n$-doped cuprate superconductors would be crucial to understanding physics of superconductivity in doped cuprates.

Theoretically, ERR has been thoroughly studied based on the phenomenological Bardeen-CooperSchrieffer (BCS) formalism with a monotonic d-wave gap and the microscopic model and understood in the $p$ doped cuprate superconductors [14 21], however, no sim- ilar studies have been made so far for the $n$-doped counterparts. In this case, an important issue is what aspects of the low energy excitations determined by ERR in both $p$-doped and $n$-doped cuprate superconductors are universal. In our recent work [22] based on the kinetic energy driven SC mechanism 23], the doping and temperature dependence of ERR in the $p$-doped cuprate superconductors has been discussed, and some main features of ERR in the $p$-doped cuprate superconductors are qualitatively reproduced [4 8]. In this paper, we study the doping and temperature dependence of ERR in the $n$-doped counterparts along with this line. We show explicitly that the pair-breaking peak energy in the $B_{2 g}$ symmetry continuously follows the $\mathrm{SC}$ transition temperature $T_{c}$ throughout the SC dome as $\omega_{\text {peak }}^{B_{2 g}} \propto T_{c}$, and therefore is a common feature for both $n$-doped and $p$-doped cuprate superconductors. However, there are pronounced deviations from a cubic response in the $B_{1 g}$ channel and a linear response in the $B_{2 g}$ channel in the low energies. Our results also show that these pronounced deviations are intriguingly related to a nonmonotonic d-wave gap in the $n$-doped cuprate superconductors.

It has been shown that the essential physics of the $n$ doped cuprate superconductors is contained in the $t-t^{\prime}-J$ model on a square lattice [3, 24],

$$
\begin{aligned}
H & =t \sum_{i \hat{\eta} \sigma} P C_{i \sigma}^{\dagger} C_{i+\hat{\eta} \sigma} P^{\dagger}-t^{\prime} \sum_{i \hat{\tau} \sigma} P C_{i \sigma}^{\dagger} C_{i+\hat{\tau} \sigma} P^{\dagger} \\
& -\mu \sum_{i \sigma} P C_{i \sigma}^{\dagger} C_{i \sigma} P^{\dagger}+J \sum_{i \hat{\eta}} \mathbf{S}_{i} \cdot \mathbf{S}_{i+\hat{\eta}},
\end{aligned}
$$

where $t<0, t^{\prime}<0, \hat{\eta}= \pm \hat{x}, \pm \hat{y}, \hat{\tau}= \pm \hat{x} \pm \hat{y}, C_{i \sigma}^{\dagger}$ $\left(C_{i \sigma}\right)$ is the electron creation (annihilation) operator, $\mathbf{S}_{i}=\left(S_{i}^{x}, S_{i}^{y}, S_{i}^{z}\right)$ are spin operators, and $\mu$ is the chemical potential. The nontrivial part of the $t-t^{\prime}-J$ model (1) resides in the projection operator $P$ which restricts the Hilbert space to exclude the zero occupancy, i.e., $\sum_{\sigma} C_{i \sigma}^{\dagger} C_{i \sigma} \geq 1$. In this case, an important question is the relation between the $p$-doped and $n$-doped cuprate superconductors. The $t-J$ model with nearest neighbor hopping $t$ has a particle-hole symmetry because the sign of $t$ can be absorbed by changing the sign of the orbital on one sublattice. However, the particle-hole asymmetry 
can be described by including the next-nearest neighbor hopping $t^{\prime}$ [25, 26]. Although there is a similar strength of the magnetic interaction $J$ for both $p$-doped and $n$-doped cuprate superconductors, the interplay of $t^{\prime}$ with $t$ and $J$ causes a further weakening of the AF spin correlation for the $p$-doped cuprate superconductors, and enhancing the AF spin correlation in the $n$-doped counterparts [25 27], therefore the AF spin correlation in the $n$-doped case is stronger than that in the $p$-doped side.

For incorporating the single occupancy constraint in the $p$-doped cuprate superconductors, we have developed a charge-spin separation (CSS) fermion-spin theory [28]. For description of the $n$-doped counterparts within this CSS fermion-spin theory, the $t-t^{\prime}-J$ model (1) can be rewritten in terms of a particle-hole transformation $C_{i \sigma} \rightarrow f_{i-\sigma}^{\dagger}$ as,

$$
\begin{aligned}
H & =-t \sum_{i \hat{\eta} \sigma} f_{i \sigma}^{\dagger} f_{i+\hat{\eta} \sigma}+t^{\prime} \sum_{i \hat{\tau} \sigma} f_{i \sigma}^{\dagger} f_{i+\hat{\tau} \sigma} \\
& +\mu \sum_{i \sigma} f_{i \sigma}^{\dagger} f_{i \sigma}+J \sum_{i \hat{\eta}} \mathbf{S}_{i} \cdot \mathbf{S}_{i+\hat{\eta}}
\end{aligned}
$$

then the local constraint $\sum_{\sigma} C_{i \sigma}^{\dagger} C_{i \sigma} \geq 1$ is transferred as $\sum_{\sigma} f_{i \sigma}^{\dagger} f_{i \sigma} \leq 1$, where $f_{i \sigma}^{\dagger}\left(f_{i \sigma}\right)$ is the hole creation (annihilation) operator. Now we follow the CSS fermion-spin theory, and decouple the hole operators as $f_{i \uparrow}=a_{i \uparrow}^{\dagger} S_{i}^{-}$ and $f_{i \downarrow}=a_{i \downarrow}^{\dagger} S_{i}^{+}$, respectively, where the spinful fermion operator $a_{i \sigma}=e^{-i \Phi_{i \sigma}} a_{i}$ represents the charge degree of freedom together with some effects of the spin configuration rearrangements due to the presence of the doped electron itself (charge carrier), while the spin operator $S_{i}$ represents the spin degree of freedom, then the single occupancy local constraint $\sum_{\sigma} f_{i \sigma}^{\dagger} f_{i \sigma} \leq 1$ is satisfied in analytical calculations. In this CSS fermion-spin representation, the $t-t^{\prime}-J$ model (2) can be expressed as,

$$
\begin{aligned}
H & =t \sum_{i \hat{\eta}}\left(a_{i+\hat{\eta} \uparrow}^{\dagger} a_{i \uparrow} S_{i}^{+} S_{i+\hat{\eta}}^{-}+a_{i+\hat{\eta} \downarrow}^{\dagger} a_{i \downarrow} S_{i}^{-} S_{i+\hat{\eta}}^{+}\right) \\
& -t^{\prime} \sum_{i \hat{\tau}}\left(a_{i+\hat{\tau} \uparrow}^{\dagger} a_{i \uparrow} S_{i}^{+} S_{i+\hat{\tau}}^{-}+a_{i+\hat{\tau} \downarrow}^{\dagger} a_{i \downarrow} S_{i}^{-} S_{i+\hat{\tau}}^{+}\right) \\
& -\mu \sum_{i \sigma} a_{i \sigma}^{\dagger} a_{i \sigma}+J_{\mathrm{eff}} \sum_{i \hat{\eta}} \mathbf{S}_{i} \cdot \mathbf{S}_{i+\hat{\eta}},
\end{aligned}
$$

where $J_{\text {eff }}=(1-\delta)^{2} J$, and $\delta=\left\langle a_{i \sigma}^{\dagger} a_{i \sigma}\right\rangle=\left\langle a_{i}^{\dagger} a_{i}\right\rangle$ is the electron doping concentration. As in the discussions of the $p$-doped case [23], the SC order parameter for the electron Cooper pair in the $n$-doped side also can be defined as,

$$
\begin{aligned}
\Delta & =\left\langle C_{i \uparrow}^{\dagger} C_{j \downarrow}^{\dagger}-C_{i \downarrow}^{\dagger} C_{j \uparrow}^{\dagger}\right\rangle=\left\langle a_{i \uparrow} a_{j \downarrow} S_{i}^{\dagger} S_{j}^{-}-a_{i \downarrow} a_{j \uparrow} S_{i}^{-} S_{j}^{+}\right\rangle \\
& =-\left\langle S_{i}^{+} S_{j}^{-}\right\rangle \Delta_{a},
\end{aligned}
$$

with the charge carrier pairing order parameter $\Delta_{a}=$ $\left\langle a_{j \downarrow} a_{i \uparrow}-a_{j \uparrow} a_{i \downarrow}\right\rangle$. It has been shown experimentally that the hot spots are located close to $[ \pm \pi, 0]$ and $[0, \pm \pi]$ (in units of inverse lattice constant) points of the Brillouin zone (BZ) in the $p$-doped case, resulting in a monotonic $\mathrm{d}$-wave gap [29]. In contrast, the hot spots are located much closer to the zone diagonal in the $n$-doped side, leading to a nonmonotonic d-wave gap [12, 30],

$$
\Delta(\mathbf{k})=\Delta\left[\gamma_{\mathbf{k}}^{(d)}-B \gamma_{\mathbf{k}}^{(2 d)}\right],
$$

where $\gamma_{\mathbf{k}}^{(d)}=\left[\cos k_{x}-\cos k_{y}\right] / 2$ and $\gamma_{\mathbf{k}}^{(2 d)}=\left[\cos \left(2 k_{x}\right)-\right.$ $\left.\cos \left(2 k_{y}\right)\right] / 2$, then the maximum gap is observed not at the BZ boundary as expected from the monotonic d-wave gap, but at the hot spot between $[\pi, 0]$ and $[\pi / 2, \pi / 2]$, where the AF spin fluctuation most strongly couples to electrons, supporting a spin-mediated pairing mechanism [30].

For a microscopic description of the SC state in cuprate superconductors, we 23] have developed a kinetic energy driven SC mechanism, where the interaction between charge carriers and spins from the kinetic energy term in the $t-t^{\prime}-J$ model (3) induces a charge carrier pairing state with the d-wave symmetry by exchanging spin excitations, while the electron Cooper pairs originating from this charge carrier pairing state are due to the chargespin recombination, and their condensation reveals the $\mathrm{SC}$ ground-state. Moreover, this SC state is a conventional BCS-like with the d-wave symmetry [31], so that some main features of the low energy electronic structure of both $p$-doped and $n$-doped cuprate superconductors have been quantitatively reproduced [31, 32]. In particular, the doping and energy evolution of the magnetic excitations in the $n$-doped cuprate superconductors has been studied [33] in terms of a nonmonotonic d-wave gap (5), and the results show that there is a broad commensurate scattering peak at low energy, then the resonance energy is located among this low energy commensurate scattering range. This low energy commensurate scattering disperses outward into a continuous ring-like incommensurate scattering at high energy. Following our previous discussions [32, 33], the full charge carrier Green function of the $n$-doped cuprate superconductors can be obtained in the Nambu representation as,

$$
g\left(\mathbf{k}, i \omega_{n}\right)=Z_{\mathrm{aF}} \frac{i \omega_{n} \tau_{0}+\bar{\xi}_{\mathrm{ak}} \tau_{3}-\bar{\Delta}_{\mathrm{aZ}}(\mathbf{k}) \tau_{1}}{\left(i \omega_{n}\right)^{2}-E_{\mathrm{ak}}^{2}},
$$

where $\tau_{0}$ is the unit matrix, $\tau_{1}$ and $\tau_{3}$ are Pauli matrices, the renormalized charge carrier excitation spectrum $\bar{\xi}_{\mathrm{ak}}=Z_{\mathrm{aF}} \xi_{\mathrm{ak}}$, with the mean-field charge carrier excitation spectrum $\xi_{\text {ak }}=Z t \chi_{1} \gamma_{\mathbf{k}}^{(s)}-Z t^{\prime} \chi_{2} \gamma_{\mathbf{k}}^{(2 s)}-\mu$, the spin correlation functions $\chi_{1}=\left\langle S_{i}^{+} S_{i+\hat{\eta}}^{-}\right\rangle$and $\chi_{2}=\left\langle S_{i}^{+} S_{i+\hat{\tau}}^{-}\right\rangle$, $\gamma_{\mathbf{k}}^{(s)}=(1 / Z) \sum_{\hat{\eta}} e^{i \mathbf{k} \cdot \hat{\eta}}, \gamma_{\mathbf{k}}^{(2 s)}=(1 / Z) \sum_{\hat{\tau}} e^{i \mathbf{k} \cdot \hat{\tau}}, Z$ is the number of the nearest neighbor or next-nearest neighbor sites, the renormalized charge carrier d-wave pair gap $\bar{\Delta}_{\mathrm{aZ}}(\mathbf{k})=Z_{\mathrm{aF}} \bar{\Delta}_{\mathrm{a}}(\mathbf{k})$, with the effective charge carrier dwave pair gap $\bar{\Delta}_{\mathrm{a}}(\mathbf{k})=\bar{\Delta}_{\mathrm{a}}\left[\gamma_{\mathbf{k}}^{(d)}-B \gamma_{\mathbf{k}}^{(2 d)}\right]$, and the charge carrier quasiparticle spectrum $E_{\mathrm{ak}}=\sqrt{\bar{\xi}_{\mathrm{ak}}^{2}+\left|\bar{\Delta}_{\mathrm{aZ}}(\mathbf{k})\right|^{2}}$, while the effective charge carrier pair gap $\bar{\Delta}_{\mathrm{a}}(\mathbf{k})$ and the quasiparticle coherent weight $Z_{\mathrm{aF}}$ satisfy the following equations [33] $\bar{\Delta}_{\mathrm{a}}(\mathbf{k})=\Sigma_{2}^{(a)}(\mathbf{k}, \omega=0)$ and 
$Z_{\mathrm{aF}}^{-1}=1-\left.\Sigma_{1 \mathrm{o}}^{(a)}(\mathbf{k}, \omega=0)\right|_{\mathbf{k}=[\pi, 0]}$, where $\Sigma_{1}^{(a)}(\mathbf{k}, \omega)$ and $\Sigma_{2}^{(a)}(\mathbf{k}, \omega)$ are the charge carrier self-energies obtained from the spin bubble, and have been given in Ref. 32 except the effective charge carrier monotonic d-wave gap has been replaced by the present nonmonotonic one, while $\Sigma_{1 \mathrm{o}}^{(a)}(\mathbf{k}, \omega)$ is the antisymmetric part of $\Sigma_{1}^{(a)}(\mathbf{k}, \omega)$. These equations have been solved simultaneously with other self-consistent equations [32, 33], then all order parameters, decoupling parameter $\alpha$, and chemical potential $\mu$ have been determined by the self-consistent calculation.

In the CSS fermion-spin representation [28], the electron Green's function,

$$
G\left(\mathbf{k}, i \omega_{n}\right)=\left(\begin{array}{ll}
G_{11}\left(\mathbf{k}, i \omega_{n}\right), & G_{12}\left(\mathbf{k}, i \omega_{n}\right) \\
G_{21}\left(\mathbf{k}, i \omega_{n}\right), & G_{22}\left(\mathbf{k}, i \omega_{n}\right)
\end{array}\right)
$$

is a convolution of the spin Green's function and charge carrier Green's function (6), and its diagonal and offdiagonal components $G_{11}\left(i-j, t-t^{\prime}\right)=\left\langle\left\langle C_{i \sigma}(t) ; C_{j \sigma}^{\dagger}\left(t^{\prime}\right)\right\rangle\right\rangle$ and $G_{21}\left(i-j, t-t^{\prime}\right)=\left\langle\left\langle C_{i \uparrow}^{\dagger}(t) ; C_{j \downarrow}^{\dagger}\left(t^{\prime}\right)\right\rangle\right\rangle$ have been given in Ref. [32].

For discussions of ERR in the $n$-doped cuprate superconductors, we need to calculate the ERR function $\tilde{S}(\mathbf{q}, \omega)$, which can be obtained in terms of the imaginary part of the Raman density-density correlation function $\tilde{\chi}(\mathbf{q}, \omega)$ as [14],

$$
\tilde{S}(\mathbf{q}, \omega)=-\frac{1}{\pi}\left[1+n_{B}(\omega)\right] \operatorname{Im} \tilde{\chi}(\mathbf{q}, \omega),
$$

with $n_{B}(\omega)$ is the boson distribution function, while the Raman density-density correlation function $\tilde{\chi}(\mathbf{q}, \omega)$ is defined as $\tilde{\chi}\left(\mathbf{q}, \tau-\tau^{\prime}\right)=-\left\langle T \rho_{\gamma}(\mathbf{q}, \tau) \rho_{\gamma}\left(-\mathbf{q}, \tau^{\prime}\right)\right\rangle$, with the Raman density operator in the Nambu representation $\rho_{\gamma}(\mathbf{q})=\sum_{\mathbf{k}} \gamma_{\mathbf{k}} C_{\mathbf{k}+\frac{\mathbf{q}}{2}}^{\dagger} \tau_{3} C_{\mathbf{k}-\frac{\mathbf{q}}{2}}$, where the bare Raman vertex $\gamma_{\mathbf{k}}$ can be classified by the representations $B_{1 g}, B_{2 g}$, and $A_{1 g}$ of the point group $D_{4 h}$ of the square lattice as [14],

$$
\gamma_{\mathbf{k}}= \begin{cases}b_{\omega_{i}, \omega_{s}}\left[\cos \left(k_{x} a\right)-\cos \left(k_{y} a\right)\right] / 4, & B_{1 g} \\ b_{\omega_{i}, \omega_{s}}^{\prime} \sin \left(k_{x} a\right) \sin \left(k_{y} a\right), & B_{2 g} \\ a_{\omega_{i}, \omega_{s}}\left[\cos \left(k_{x} a\right)+\cos \left(k_{y} a\right)\right] / 4, & A_{1 g}\end{cases}
$$

respectively, where as a qualitative discussion, the magnitude of the energy dependence of the prefactors $b, b^{\prime}$ and $a$ can be rescaled to units. The Raman vertex $\gamma_{\mathbf{k}}$ (9) also shows that for the $B_{2 g}$ channel, the Raman vertex is most sensitive to the nodal $([ \pm \pi / 2, \pm \pi / 2])$ region of the $\mathrm{BZ}$ and vanishes along $[0,0] \rightarrow[\pi, 0]$ and equivalent lines of the BZ. However, for the $B_{1 g}$ channel, the nodal $[0,0] \rightarrow[\pi, \pi]$ diagonals do not contribute to the intensity that mainly integrates from regions near intersections of the Fermi surface and the BZ boundary. In this case, the Raman density-density correlation function $\tilde{\chi}(\mathbf{q}, \omega)$ can be expressed in terms of the electron Green's function (7) as,

$$
\begin{aligned}
\tilde{\chi}_{\gamma_{1} \gamma_{2}}\left(\mathbf{q}, i q_{n}\right) & =\frac{1}{N} \sum_{\mathbf{k}} \gamma_{1 \mathbf{k}} \gamma_{2 \mathbf{k}} \frac{1}{\beta} \sum_{i \omega_{n}} \operatorname{Tr}\left[G\left(\mathbf{k}-\mathbf{q} / 2, i \omega_{n}\right)\right. \\
& \left.\times \tau_{3} G\left(\mathbf{k}+\mathbf{q} / 2, i \omega_{n}-i q_{n}\right) \tau_{3}\right],
\end{aligned}
$$

then the explicit form of the obtained $\tilde{\chi}_{\gamma_{1} \gamma_{2}}(\mathbf{q}, \omega)$ in the present $n$-doped cuprate superconductors is the same as that given in Ref. 22 for the $p$-doped counterparts except the charge carrier quasiparticle coherence factors $U_{1 \mathbf{h k}}^{2}=\left(1+\overline{\xi_{\mathbf{k}}} / E_{\mathrm{hk}}\right) / 2$ and $U_{2 \mathrm{~h} \mathbf{k}}^{2}=\left(1-\overline{\xi_{\mathbf{k}}} / E_{\mathrm{hk}}\right) / 2$, the renormalized charge carrier d-wave pair gap $\bar{\Delta}_{\mathrm{hZ}}(\mathbf{k})$, and the charge carrier quasiparticle spectrum $E_{\mathrm{hk}}$ have been replaced as $U_{\text {1ak }}^{2}=\left(1-\overline{\xi_{\mathbf{k}}} / E_{\mathrm{ak}}\right) / 2$ and $U_{2 \mathrm{ak}}^{2}=$ $\left(1+\overline{\xi_{\mathbf{k}}} / E_{\mathrm{ak}}\right) / 2, \bar{\Delta}_{\mathrm{aZ}}(\mathbf{k})$, and $E_{\mathrm{ak}}$, respectively. This result shows that the Raman density-density correlation function (10) [then the ERR function (8)] is closely related to the pair coherence factors, which give rise to the occurrence of the pair-breaking features in the spectra [14, 22]. The above Raman density-density correlation function (101) also shows that the variation with momentum of the Raman vertex $\gamma_{\mathbf{k}}$ is coupled to the momentum dependence of the charge carrier nonmonotonic d-wave gap $\bar{\Delta}_{\mathrm{aZ}}(\mathbf{k})$ in the $n$-doped case, leading to a strong polarization dependence of the spectra.

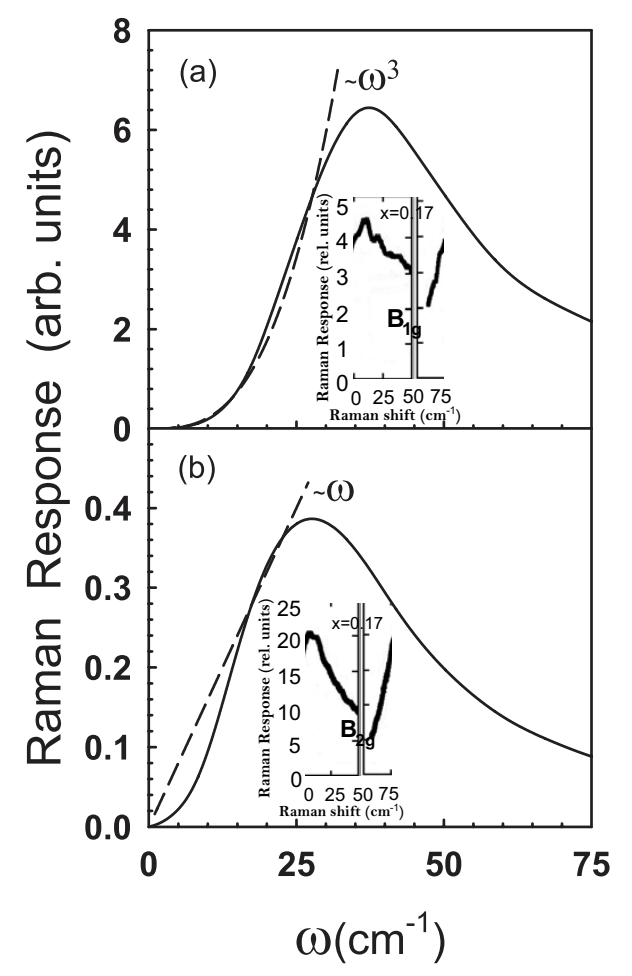

FIG. 1: (a) $B_{1 g}$ and (b) $B_{2 g}$ spectra as a function of energy at $p=0.17$ for $T=3 \mathrm{~K}$. The dashed lines are a cubic and a linear fit for the low-energy $B_{1 g}$ and $B_{2 g}$ spectra, respectively. Inset: the corresponding experimental results of $\operatorname{Pr}_{2-x} \mathrm{Ce}_{x} \mathrm{CuO}_{4-\delta}$ taken from Ref. 13.

For the $n$-doped cuprate superconductors, although the values of $J, t$, and $t^{\prime}$ in the $t-t^{\prime}-J$ model (1) are be- 
lieved to vary somewhat from compound to compound, the numerical calculations [25, 26] have extracted the range of these parameters as $J \approx 0.1 \sim 0.13 \mathrm{eV}, t / J=$ $-2.5 \sim-3$, and $t^{\prime} / t$ is of order $0.2 \sim 0.3$. However, as a qualitative discussion in this paper, the commonly used parameters are chosen as $t / J=-2.5, t^{\prime} / t=0.3$, and $J=0.13 \mathrm{eV} \approx 1500 \mathrm{~K} \approx 1049 \mathrm{~cm}^{-1}$. We are now ready to discuss the doping and temperature dependence of ERR in the $n$-doped cuprate superconductors. We have performed a calculation for the ERR function (8) in both $B_{1 g}$ and $B_{2 g}$ orientations, and the results of (a) the $B_{1 g}$ and (b) $B_{2 g}$ spectra at the overdoping $p=0.17$ in temperature $T=3 \mathrm{~K}$ are plotted in Fig. 1 in comparison with the corresponding experimental results [13] of the $n$-doped cuprate superconductor $\mathrm{Pr}_{2-x} \mathrm{Ce}_{x} \mathrm{CuO}_{4-\delta}(\mathrm{PCCO})$ are also presented in Fig. 11(inset). As in the hole-doped case 22], both $B_{1 g}$ and $B_{2 g}$ spectra in the overdoped regime are characterized by the presence of the pair-breaking $(2 \Delta)$ peaks and low-energy tails. These low-energy tails of the Raman continuum change to reflect the opening of the gap, i.e., the strength of the low-energy continuum is reduced and the spectrum acquires the pair-breaking peak as a result of excitations across the anisotropic gap $2 \Delta(\mathbf{k})$, while the pair-breaking peaks correspond to the excitations out of the SC condensate. However, for different scattering geometries spectra differ in their intensity as well as in the position of the $2 \Delta$ peaks. Although the effective charge carrier pair gap $\bar{\Delta}_{\mathrm{aZ}}(\mathbf{k})$ [then the SC gap (50) obviously deviates from the monotonic d-wave gap [30], it is basically consistent with the d-wave symmetry. This leads to that the pair-breaking peak intensities in both $B_{1 g}$ and $B_{2 g}$ below the SC coherence peaks vanish smoothly without a threshold to zero energy [22]. Furthermore, we have also fitted the low-energy tails to an $\omega^{3}$ power law in the $B_{1 g}$ response and linearly with $\omega$ in the $B_{2 g}$ response, however, in sharp contrast to the $p$-doped case [22], there are pronounced deviations from a cubic response in the $B_{1 g}$ channel and a linear response in the $B_{2 g}$ channel. For the $p$-doped cuprate superconductors, the interpretation of the cubic response in the $B_{1 g}$ channel and linear response in the $B_{2 g}$ channel is consistent with the monotonic d-wave gap [14, 22]. In comparison with the $p$-doped case, our present results also show that these pronounced deviations in the low energies in the $n$-doped counterparts are mainly caused by the nonmonotonic d-wave gap, which is also consistent with the interpretation in terms of the phenomenological BCS formalism with the nonmonotonic d-wave gap given in Ref. 12. However, there is a substantial difference between theory and experiment, namely, in the extremely low energies, the calculated ERR spectrum in the $B_{1 g}$ channel is roughly consistent with an $\omega^{3}$ power law, while the calculated ERR spectrum in the $B_{2 g}$ channel is in disagreement with a linear $\omega$ response. This result in the extremely low energies seems not to be consistent with the corresponding experimental data [13], where in the extremely low energies, the ERR spectrum in the $B_{1 g}$ channel does not show an $\omega^{3}$ behavior, while the ERR spectrum in the $B_{2 g}$ channel may be a linear $\omega$ dependence. The simple $t-J$ model can not be regarded as a complete model for the quantitative comparison with cuprate superconductors, however, as a qualitative discussion in this paper, the overall shape seen in the theoretical result is qualitatively consistent with that observed in the experiment [13].

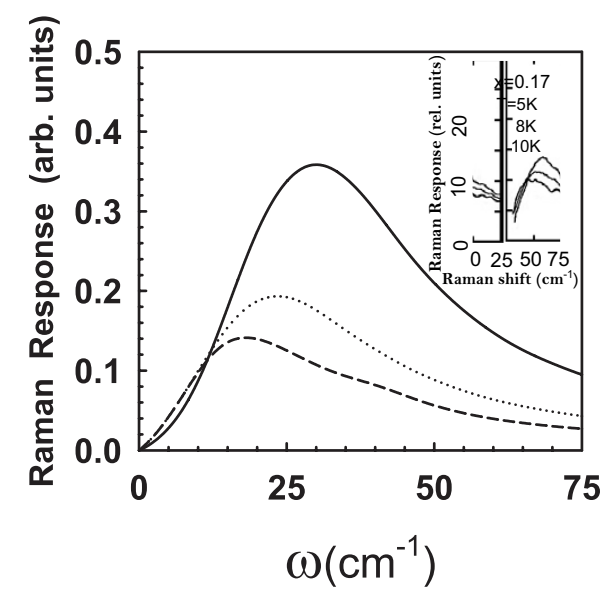

FIG. 2: $B_{2 g}$ spectrum as a function of energy at $p=0.17$ for $T=5.2 \mathrm{~K}$ (solid line), $T=8.2 \mathrm{~K}$ (dotted line), and $T=10.5 \mathrm{~K}$ (dashed line). Inset: the corresponding experimental results of $\mathrm{Pr}_{2-x} \mathrm{Ce}_{x} \mathrm{CuO}_{4-\delta}$ taken from Ref. 13.

ERR in Fig. 1 is also temperature dependent. To analyze the evolution of ERR in Fig. 1 with temperature, we have performed a calculation for the ERR function (8) in the $B_{2 g}$ channel with different temperatures, and the results of the $B_{2 g}$ spectrum as a function of energy with $T=5.2 \mathrm{~K}$ (solid line), $T=8.2 \mathrm{~K}$ (dotted line), and $T=10.5 \mathrm{~K}$ (dashed line) for $p=0.17$ are plotted in Fig. 2 in comparison with the corresponding experimental results [13] of PCCO (inset). Within the kinetic energy driven SC mechanism, the calculated SC transition temperature $T_{c}=33 \mathrm{~K}$ at $p=0.17$. This anticipated SC transition temperature is not too far from the SC transition temperature $T_{c}=13 \mathrm{~K}$ observed experimentally on PCCO at $p=0.17$ [13]. Obviously, the experimental results of the ERR spectrum [13] are qualitatively reproduced. In particular, the pair-breaking peak loses intensity and moves to lower energies by increasing temperatures. Furthermore, as in the $p$-doped case [22], this pair-breaking peak intensity in the $n$-doped counterparts also follows a pair gap type temperature dependence, and disappears at the SC transition temperature $T_{c}$.

Now we turn to discuss the doping dependence of ERR. We have made a series of calculations for the ERR function (8) with different doping concentrations, and the results of the (a) $B_{1 g}$ and (b) $B_{2 g}$ spectra as a function of energy with $p=0.165$ (solid line) and $p=0.17$ (dashed line) at $T=3 \mathrm{~K}$ are plotted in Fig. 3 in comparison with the corresponding experimental results [13] of PCCO (inset). Our results show that with increasing the doping concentration in the overdoped regime, the 


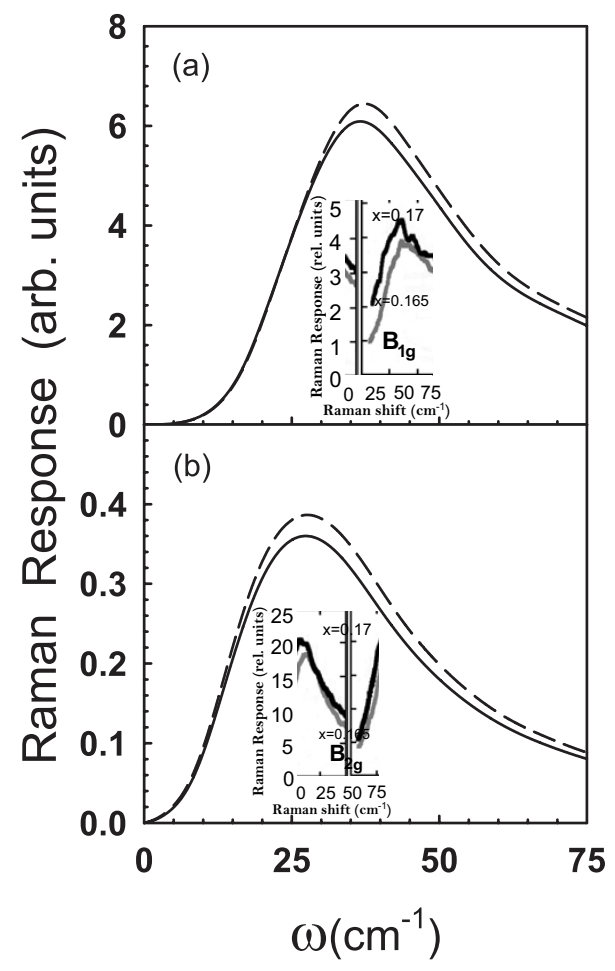

FIG. 3: (a) $B_{1 g}$ and (b) $B_{2 g}$ spectra as a function of energy at $p=0.165$ (solid line) and $p=0.17$ (dashed line) for $T=3 \mathrm{~K}$. Inset: the corresponding experimental results of $\mathrm{Pr}_{2-x} \mathrm{Ce}_{x} \mathrm{CuO}_{4-\delta}$ taken from Ref. 13 .

weights of the peaks in both $B_{1 g}$ and $B_{2 g}$ symmetries increase, while the peak energies in both $B_{1 g}$ and $B_{2 g}$ channels decrease. However, since there is the weakness of the calculated ERR spectrum in the extremely low energies as shown in Fig. 1, the doping dependence of the ERR spectrum in the low energies is better than that in the extremely low energies. Moreover, in contrast to the case in the overdoped regime, the peak energies in both $B_{1 g}$ and $B_{2 g}$ channels move to the higher energies when increasing doping concentration in the underdoped regime. To show this point clearly, we have calculated the ERR function (8) throughout the SC dome, and then employed the shift of the leading-edge mid-point as a measurement of the magnitude of the gap at each doping concentration just as it has been done in the experiments [13]. The results for the extracted (a) $B_{1 g}$ and (b) $B_{2 g}$ peak energies as a function of doping with $T=3 \mathrm{~K}$ are plotted in Fig. 4. For comparison, the corresponding experimental results [13] of PCCO are also presented in Fig. 4 (inset). It is shown that both peak energies have pronounced maximum around the optimal doping, and then decreases in both underdoped and overdoped regimes. The maximum values of the peak energy for the $B_{2 q}$ channel throughout the SC dome are very similar to the single particle spectroscopy gap values [3, 32], and therefore continuously follows the SC transition temperature as $\omega_{\text {peak }}^{B_{2 g}} \propto T_{c}$. In this sense, it can be closely

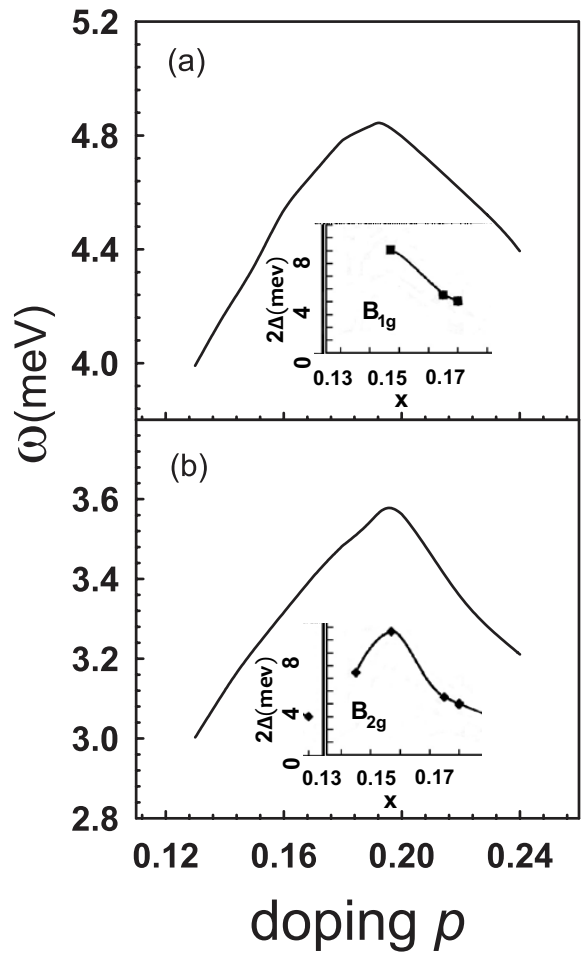

FIG. 4: (a) $B_{1 g}$ and (b) $B_{2 g}$ peaks as a function of doping for $T=3 \mathrm{~K}$. Inset: the corresponding experimental results of $\operatorname{Pr}_{2-x} \mathrm{Ce}_{x} \mathrm{CuO}_{4-\delta}$ taken from Ref. 13.

associated with twice the gap magnitude, in good agreement with the experimental results [13]. Furthermore, incorporating with our previous results of ERR for the $p$-doped case [22], our present results also show that the domelike shape of the doping dependent peak energy in the $B_{2 g}$ symmetry is a common feature for both electrondoped and hole-doped cuprate superconductors. In the $B_{1 g}$ orientation, our present results of the maximum values of the peak energy in the optimally and overdoped regimes are in qualitative agreement with the the corresponding experimental results [13] of PCCO. This seems to show that peak energy in $B_{1 g}$ channel in the optimally and overdoped regimes is qualitatively related to the gap. However, in the underdoped regime, no extracted maximum values of the Raman scattering peak energy from experimental measurements on the $n$-doped counterparts are available now. In this case, we can not make a direct comparison for the present theoretical results with the corresponding experimental data in the underdoped regime, and therefore present theoretical results in the underdoped regime need to be verified by further Raman scattering experiments. On the other hand, since the integrated reduced coherence peak intensity in the SC state is proportional to the superfluid density $\rho_{s}$ [13], therefore the domelike shape of the doping dependent ERR spectrum in the $n$-doped cuprate superconductors implies that the superfluid density $\rho_{s}$ increases with increasing doping in the lower doped regime, and reaches 
a maximum around the critical doping, then decreases in the higher doped regime. This has been confirmed by our study of the doping dependence of the superfluid density in the $n$-doped cuprate superconductors [34].

The electronic states in solids are characterized by their energy dispersions as well as the characteristic lifetime of an electron placed into such a state. This state is represented by the single-particle propagator, while the spectral function is directly related to the analytically continued single-particle propagator [14]. In particular, this spectral function is measurable via angle-resolved photoemission spectroscopy (ARPES) techniques and can provide an important information about quasiparticles [3]. Within the framework of the kinetic energy driven SC mechanism, the essential physics of ERR in the $n$-doped cuprate superconductors is the same as that in the $p$-doped counterparts 22]. The kinetic energy driven SC-state in the $n$-doped cuprate superconductors is the conventional BCS like with the d-wave symmetry [32], where it has been shown that this kinetic energy driven d-wave BCS formalism can reproduce quantitatively some main low energy features of the ARPES experimental data of the $n$-doped cuprate superconductors. However, many of the unusual physical properties of the two-particle electron dynamics across the phase diagram are attributed to particular characteristics of low energy excitations determined by ERR. Although the differences between single-particle and two-particle properties are inescapable in cuprate superconductors, the single-particle and two-particle correlation functions can be related each other [14]. This is why the kinetic energy driven d-wave BCS formalism is still valid in discussions of the doping and temperature dependence of ERR in the $n$-doped cuprate superconductors.

In the $p$-doped cuprate superconductors, the Raman scattering experimental data show that the peak energy in $B_{1 g}$ channel increases essentially linearly as the doping concentration is reduced in the underdoped regime [7, 8], which reflects that the peak energy in the $B_{1 g}$ symmetry is progressively disconnected from superconductivity as one goes from the overdoped regime to the underdoped regime. In Ref. 8 , it has been argued that for a correct description of the $B_{1 g}$ spectrum in the $p$-doped cuprate superconductors in the underdoped regime, two essential ingredients should be taken into account within the d-wave BCS formalism: (1) the quasiparticle spectral weight $Z_{\mathrm{F}}(\mathbf{k})$ as well as the vertex correction describing the interaction of the quasiparticles with external perturbations; and (2) a general gap also should be taken into account by including the higher order of the harmonic component in the monotonic d-wave gap. In our recent work based on the monotonic d-wave gap for the $p$-doped cuprate superconductors [22], the first condition is partially satisfied, since the doping dependence of the quasiparticle spectral weight $Z_{\mathrm{F}}$ has been included in the discussions. However, the vertex correction for the interaction of the quasiparticles due to the presence of the spin fluctuation is not included, which also is doping and momentum dependent. In this case, our theoretical results 22] of ERR for the $p$-doped cuprate superconductors in the $B_{1 g}$ channel in the underdoped regime is in disagreement with the corresponding experimental data 7, [8]. For the second condition, our present study shows indirectly that if the vertex correction for the interaction of the quasiparticles with external perturbations is not considered, even a more general nonmonotonic d-wave gap (5) is used for discussions of ERR in the $p$-doped cuprate superconductors, the correct behavior of ERR in the $B_{1 g}$ channel in the underdoped regime still can not be reproduced. Thus an important issue is how the vertex correction for the interaction of the quasiparticles due to the presence of the spin fluctuation is taken into account within the kinetic energy driven d-wave BCS formalism for a correct description of the $B_{1 g}$ spectrum of the $p$-doped cuprate superconductors in the underdoped regime. On the other hand, the effect of a weak magnetic field on ERR is not considered in the present work. However, the depairing due to the Pauli spin polarization is very important in the presence of a weak magnetic field, since both $p$-doped and $n$-doped cuprate superconductors are doped Mott insulators with the strong short-range $\mathrm{AF}$ correlation dominating the entire $\mathrm{SC}$ phase [3]. In particular, in the kinetic energy driven SC mechanism [23], where the charge carrier-spin interaction from the kinetic energy term induces a d-wave pairing state by exchanging spin excitations. Therefore under the kinetic energy driven SC mechanism, an external magnetic field aligns the spins of the unpaired electrons, then the dwave electron Cooper pairs in both $p$-doped and $n$-doped cuprate superconductors can not take advantage of the lower energy offered by a spin-polarized state. This leads to that a weak magnetic field may suppress the pairbreaking peak magnitude in the ERR spectrum at a rapid rate [13, 35]. These and the related issues are under investigation now.

In summary, we have studied the doping and temperature dependence of ERR in the $n$-doped cuprate superconductors based on the kinetic energy driven SC mechanism. Our results show that the pair-breaking peak energy in the $B_{2 g}$ symmetry continuously follows the SC transition temperature $T_{c}$ throughout the $\mathrm{SC}$ dome as $\omega_{\text {peak }}^{B_{2 g}} \propto T_{c}$, and therefore is a common feature for both $n$-doped and $p$-doped cuprate superconductors. However, in sharp contrast to the $p$-doped counterparts, there are pronounced deviations from a cubic response in the $B_{1 g}$ channel and a linear response in the $B_{2 g}$ channel in the low energies. Our results also show that these pronounced deviations are mainly caused by a nonmonotonic d-wave gap in the $n$-doped cuprate superconductors.

Within the framework of the kinetic energy driven $\mathrm{SC}$ mechanism, the electromagnetic response in both $p$ doped and $n$-doped cuprate superconductors in the SC state has been studied [34 36]. It is shown that the magnetic field penetration depth shows a crossover from the linear temperature dependence at low temperatures to a nonlinear one at the extremely low temperatures. In 
particular, in analogy to the domelike shape of the doping dependent SC transition temperature, the maximal superfluid density occurs around the critical doping, and then decreases in both lower doped and higher doped regimes. These are consistent with the present results of the Raman response in the $n$-doped cuprate superconductors and our recent results 22] of the Raman response in the $p$-doped cuprate superconductors, and all these studies are important to confirm the nature of the SC phase of both $p$-doped and $n$-doped cuprate superconductors as a d-wave BCS-like SC state within the kinetic energy driven SC mechanism.

\section{Acknowledgments}

The authors would like to thank Dr. Zheyu Huang for helpful discussions. This work was supported by the National Natural Science Foundation of China under Grant No. 11074023, and the funds from the Ministry of Science and Technology of China under Grant No. 2011CB921700.
[1] J. G. Bednorz and K. A. Müller, Z. Phys. B 64 (1986) 189.

[2] Y. Tokura, H. Takagi, and S. Uchida, Nature 337 (1989) 345 .

[3] A. Damascelli, Z. Hussain, and Z. X. Shen, Rev. Mod. Phys. 75 (2003) 473.

[4] X. K. Chen, J. C. Irwin, H. J. Trodahl, M. Okuya, T. Kimura, and K. Kishio, Physica C 295 (1998) 80.

[5] W. Guyard, A. Sacuto, M. Cazayous, Y. Gallais, M. LE Tacon, D. Colson and A. Forget, Phys. Rev. Lett 101 (2008) 097003.

[6] F. Venturini, M. Opel, R. Hackl, H. Berger, L. Forró, B. Revaz, J. Phys. Chem. Solids 63 (2002) 2345.

[7] W. Guyard, M. LE Tacon, M. Cazayous, A. Sacuto, A. Georges, D. Colson and A. Forget, Phys. Rev. B 77 (2008) 024524.

[8] M. LE Tacon, A. Sacuto, A. Georges, G. Kotliar, Y. Gallais, D. Colson and A. Forget, Nature Physics. 2 (2006) 537.

[9] N. P. Armitage, P. Fournier, and R. L. Greene, Rev. Mod. Phys. 82 (2010) 2421.

[10] B. Stadlober, G. Krug, R. Nemetschek, and R. Hackl, J. L. Cobb, and J. T. Markert, Phys. Rev. Lett. 74 (1995) 4911.

[11] C. Kendziora, B. Nachumi, P. Fournier, Z. Y. Li, R. L. Greene, D. G. Hinks, Physica C 364-365 (2001) 541.

[12] G. Blumberg, A. Koitzsch, A. Gozar, B. S. Dennis, C. A. Kendziora, P. Fournier, and R. L. Greene Phys. Rev. Lett. 88 (2002) 107002.

[13] M. M. Qazilbash, A. Koitzsch, B. S. Dennis, A. Gozar, Hamza Balci, C. A. Kendziora, R. L. Greene, and G. Blumberg, Phys. Rev B 72 (2005) 214510.

[14] T. P. Devereaux and R. Hackl, Rev. Mod. Phys. 79 (2007) 175, and references therein.

[15] R. Zeyher and A. Greco, Phys. Rev. Lett. 89 (2002) 177004 .

[16] T. P. Devereaux, D. Einzel, B. Stadlober, R. Hackl, D. H. Leach, and J. J. Neumeier, Phys. Rev. Lett. 72 (1994) 396; D. Branch and J. P. Carbotte, Phys. Rev. B 54 (1996) 13288.

[17] D. Branch and J. P. Carbotte, Phys. Rev. B 52 (1995) 603.

[18] D. Manske, C. T. Rieck, R. Das Sharma, A. Bock, and
D. Fay, Phys. Rev. B 56 (1997) R2940.

[19] T. Dahm, D. Manske, and L. Tewordt, Phys. Rev. B 58 (1998) 12454.

[20] O. V. Misochko and E. Ya Sherman, J. Phys.: Condens. Matter 12 (2000) 9095; E. Ya Sherman, C. AmbroschDraxl, and O. V. Misochko, Phys. Rev. B 65 (2002) $140510(\mathrm{R})$.

[21] A. V. Chubukov, T. P. Devereaux, and M. V. Klein, Phys. Rev. B 73 (2006) 094512.

[22] Zhihao Geng and Shiping Feng, Phys. Lett. A 375 (2010) 214.

[23] Shiping Feng, Phys. Rev. B 68 (2003) 184501; Shiping Feng, Tianxing Ma, and Huaiming Guo, Physica C 436 (2006) 14.

[24] P. W. Anderson, Science 235 (1987) 1196.

[25] M.S. Hybertson, E.B. Stechel, M. Schuter, and D.R. Jennison, Phys. Rev. B 41 (1990) 11068.

[26] E. Pavarini, I. Dasgupta, T. Saha-Dasgupta, O. Jepsen, and O. K. Andersen, Phys. Rev. Lett. 87 (2001) 47003.

[27] R.J. Gooding, K.J.E. Vos, and P.W. Leung, Phys. Rev. B 50 (1994) 12866.

[28] Shiping Feng, Jihong Qin, and Tianxing Ma, J. Phys. Condens. Matter 16 (2004) 343.

[29] H. Ding, M. R. Norman, J. C. Campuzano, M. Randeria, A. F. Bellman, T. Yokoya, T. Takahashi, T. Mochiku, and K. Kadowaki, Phys. Rev. B 54 (1996) R9678.

[30] H. Matsui, K. Terashima, T. Sato, T. Takahashi, M. Fujita, and K. Yamada, Phys. Rev. Lett. 95 (2005) 017003.

[31] Huaiming Guo and Shiping Feng, Phys. Lett. A 361 (2007) 382; Yu Lan, Jihong Qin, and Shiping Feng, Phys. Rev. B 76 (2007) 014533; Weifang Wang, Zhi Wang, Jingge Zhang, and Shiping Feng, Phys. Lett. A 374 (2010) 632.

[32] Li Cheng, Huaiming Guo, and Shiping Feng, Phys. Lett. A 366 (2007) 137.

[33] Li Cheng and Shiping Feng, Phys. Rev B 77 (2008) 054518.

[34] Zheyu Huang and Shiping Feng, unpublished.

[35] Zheyu Huang, Huaisong Zhao, and Shiping Feng, Phys. Rev B 83 (2011) 144524.

[36] Shiping Feng, Zheyu Huang, and Huaisong Zhao, Physica C 470 (2010) 1968. 